\title{
Selección Espermática en Semen Congelado/Descongelado de Equino: Evaluación de las Membranas Plasmática, Acrosomal y Potencial de Membrana Mitocondrial
}

\author{
Sperm Selection in Frozen/Thawed Semen of Equine: Evaluation of Plasma, \\ Acrosome Membranes and Mitochondrial Membrane Potential
}

Paulina Cabrera*; Raúl Sánchez ${ }^{*, * *}$ \& Jennie Risopatrón",****

CABRERA, P.; SÁNCHEZ, R. \& RISOPATRÓN, J. Selección espermática en semen congelado/descongelado de equino: evaluación de las membranas plasmática, acrosomal y potencial de membrana mitocondrial. Int. J. Morphol., 32(2):725-731, 2014.

RESUMEN: Los procedimientos de criopreservación inducen cambios morfofuncionales en los espermatozoides. Es importante post descongelación espermática utilizar procedimientos de selección que permitan recuperar espermatozoides altamente funcionales. El objetivo del presente estudio fue comparar la eficiencia del Swim-up y Equipure $\AA^{\circledR}$ en la selección de espermatozoides funcionales en semen descongelado de equino. Semen de 4 potros reproductores Criollos Chilenos (A, B, C y D), fueron descongelados separadamente y procesados (n=15) por: I.- Swim-up (SU) y II.- Equipure ${ }^{\circledR}(E Q)$. Post descongelación se determinó por citometría de flujo la viabilidad e integridad de membrana plasmática (SYBR-14/PI), potencial de membrana mitocondrial ( $\Psi \Delta \mathrm{m}$; JC-1), integridad de la membrana acrosomal (FITC-PSA/PI). La motilidad progresiva (\%) en dos animales fue más alta $(\mathrm{P}<0,05)$ por SU comparado con EQ: A $(55,7 \pm 5,8 \%$ v/s $38,17 \pm 3,7 \%)$ y C $(37,5 \pm 7 \%$ vs. $32 \pm 2,1 \%$, respectivamente). La integridad de la membrana plasmática (\%), tres animales presentaron diferencias $(\mathrm{P}<0,05)$, siendo más alta por $\mathrm{SU}$ en dos animales comparado con EQ (A: $54,3 \pm 1,7$ vs. 36,7 $\pm 1,9$, C: $36,1 \pm 5,7$ vs. $29,4 \pm 4,8$ y D: $34,4 \pm 9,4$ vs. $52,7 \pm 5,2$; respectivamente), solamente un animal fue superior EQ. En el $\Psi \Delta \mathrm{m}(\%)$, diferencias significativas $(\mathrm{P}<0,05)$

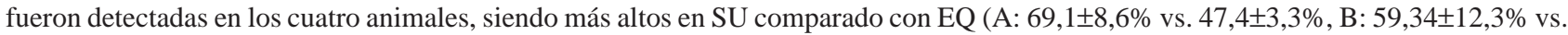
$24,8 \pm 1,5 \%$, C: $54,9 \pm 12,3 \%$ vs. $43,2 \pm 3,1 \%$ y D: $53,1 \pm 17,6 \%$ vs. $37,5 \pm 5,7 \%$; respectivamente). Los resultados obtenidos en el presente estudio demostraron que los métodos de selección espermática Swim-up y Equipure ${ }^{\circ}$ permiten recuperar espermatozoides de diferente calidad funcional en semen congelado-descongelado de equino, presentándose diferencias individuales entre los animales con respecto a los métodos. Se observó una tendencia del método Swim-up en seleccionar espermatozoides de equino descongelados con mayor calidad funcional comparado con Equipure®.

PALABRAS CLAVE. Equino; Espermatozoide; Swim-up; Equipure®

\section{INTRODUCCIÓN}

La aplicación de técnicas de asistencia reproductiva tales como inseminación artificial (IA) con semen congelado permiten aumentar el potencial reproductivo de los equinos (Pojprasath et al., 2011). La criopreservación de espermatozoides es de gran relevancia para la industria de reproducción equina, permitiendo el almacenamiento por períodos de tiempo prolongados y el transporte de semen a lugares distantes (Hoffmann et al., 2011) y utilizar a los potros simultáneamente en reproducción y en competición (Schober et al., 2007). A pesar de la creciente demanda comercial para utilizar semen congelado de equino, la tasa de preñez después de la IA es más baja comparadas con las obtenidas con semen fresco o refrigerado, e incluso inferiores a la obtenida en otras especies domésticas (LópezFernández et al., 2007). Es importante señalar que, existe un alto grado de variabilidad en la calidad del semen entre los machos e incluso entre los eyaculados de un mismo animal (Dowsett \& Knott, 1996) como también en resistir los procesos de congelación-descongelación, en términos de supervivencia de los espermatozoides después de la descongelación (Hoffmann et al.). Es conocido que los procedimientos de congelación y descongelación inducen una serie de efectos nocivos en las células espermáticas y consecuentemente una baja tasa de preñez. En equinos, se ha demos-

\footnotetext{
* Centro de Biotecnología en Reproducción (CEBIOR- BIOREN), Facultad de Medicina, Universidad de La Frontera, Temuco, Chile.

** Departamento de Ciencias Preclínicas, Facultad de Medicina, Universidad de La Frontera, Temuco, Chile.

**** Departamento de Ciencias Básicas, Facultad de Medicina, Universidad de La Frontera, Temuco, Chile.
} 
trado que inducen fragmentación del ADN espermático (Baumber et al., 2003), disminución del $\Psi \Delta \mathrm{m}$ (Brum et al., 2008), daños en el acrosoma (Atroshchenkoa et al., 2011) y generación de ROS espermático (anión superóxido intracelular) (Burnaugh et al., 2007).

En la etapa de post-descongelación es importante utilizar procedimientos de selección de espermatozoides que permitan recuperar una población espermática altamente funcional, condiciones indispensables para lograr tasas óptimas de concepción. En equinos, varias técnicas han sido utilizadas, incluyendo las basadas en separación por columnas (Sephadex y lana de vidrio) y por gradiente de densidad (Sieme et al., 2003; Stuhtmann et al., 2012). Previos estudios en espermatozoides descongelados de equino, han demostrado que el Equipure (Stoll et al., 2013) y el procedimiento de Swim-up (Sieme et al., 2003), permiten la separación de espermatozoides con morfología normal y alta motilidad. En presente estudio se evalúo la eficacia de Swim-up y Equipure® en la recuperación de espermatozoides descongelados de equino de alta calidad con respecto a la integridad de las membranas plasmática, acrosomal y $\Psi \Delta \mathrm{m}$.

\section{MATERIAL Y MÉTODO}

Animales. Se utilizaron 4 potros criollos chilenos con edades entre 7-18 años. Los animales permanecieron en el Criadero Santa Teresa, Caivico, IX Región de la Araucanía, Chile y fueron controlados periódicamente por un Médico Veterinario, mantenidos con una dieta balanceada en pesebrera y libre acceso al agua.

Colección y transporte del semen La colección de las muestras de semen se realizó durante la temporada reproductiva septiembre-marzo (Ortega Ferrusola et al., 2010). El eyaculado de cada potro fue obtenido a través de estimulación con una yegua en celo (Foster et al., 2011) y el semen fue recolectado en una vagina artificial tipo colorado a de $40-50^{\circ} \mathrm{C}$. Posteriormente, fue filtrado y diluido $(1: 3 \mathrm{v} /$ v) en un medio de transporte (Marcias Garcia et al., 2011) en tubos Falcon de $50 \mathrm{ml}$ y transportado al laboratorio del CEBIOR a una temperatura aproximada de $35^{\circ} \mathrm{C}$. El examen microscópico inmediato del semen se realizó por observación directa de $10 \mu \mathrm{l}$ de muestra depositada en un portaobjeto y cubierto con un cubre objeto de $22 \times 22$, en un microscopio de luz óptica (Axiolab drd, KT 450905, Zeiss) con aumento de 400x. Se categorizó el movimiento espermático de acuerdo a descrito previamente por Brinsko et al. (2011) en: a) Motilidad total, que es el porcentaje de espermatozoides que tienen cualquier tipo de movimiento y b) Motilidad progresiva, porcentaje de espermatozoides que exhiben un movimiento lineal y rápido. En el estudio solo se utilizaron muestras con motilidad progresiva $>60 \%$ (Marcías-García et al.).

Medios. I. Medio de transporte (MT). Para el trasporte se utilizó el medio compuesto por $2,4 \mathrm{~g}$ de leche descremada, 4,9 g de glucosa, $2 \mathrm{ml}$ de $\mathrm{NaHCO} 3,100,000$ IU de penicilina $\mathrm{G}$ sódica, $10 \mathrm{mg}$ de estreptomicina, $98 \mathrm{ml}$ de agua desionizada, $\mathrm{pH} 7,35 ; 350$ mOsm (Pojprasath et $a l$. .). II. Medio de congelación (MC): A $25 \mathrm{ml}$ medio de transporte se le adicionó $20 \mathrm{ml}$ yema de huevo, 0,5 ml Equex STM paste [Nova Chemical Sales, Scituate, MA, USA], $50 \mathrm{ml} \mathrm{lactosa}$ al 11\%. pH 7,0; 300 mOsm (Pojprasath et al.). III. Medio modificado de Tyrode (TALP): $72 \mathrm{mM}$ $\mathrm{NaCl}, 25 \mathrm{mM} \mathrm{KCl}, 25 \mathrm{mM} \mathrm{NaHCO} 3,0,36 \mathrm{mM} \mathrm{NaH} 2 \mathrm{PO} 4$, $2 \mathrm{mM} \mathrm{CaCl}-2 \mathrm{H} 2 \mathrm{O}, 0,4 \mathrm{mM} \mathrm{MgCl}-6 \mathrm{H} 2 \mathrm{O}, 25 \mathrm{mM}$ HEPES, $5 \mathrm{mM}$ glucosa, 21,6 mM lactato de sodio y $0,25 \mathrm{mM}$ piruvato de sodio, $\mathrm{pH}$ 7,4 (Brum et al.).

Congelación y descongelación del semen. En cada ensayo experimental $(\mathrm{n}=15)$, la congelación del semen de cada uno de los potros se realizó de acuerdo a lo descrito previamente por Ortega Ferrusola et al. El semen fue centrifugado a $600 \mathrm{x}$ g por $10 \mathrm{~min}$, el plasma seminal eliminado y el pellet diluido en MC para obtener una concentración ajustada a 200 x $10^{6}$ espermatozoides $/ \mathrm{ml}$, a temperatura ambiente. Posteriormente, la suspensión espermática fue enfriada gradualmente hasta $\operatorname{los} 4^{\circ} \mathrm{C}$ durante 1 hora $\left(0,3{ }^{\circ} \mathrm{C} / \mathrm{min}\right)$ y almacenadas en pajuelas de $0,5 \mathrm{ml}$ a una concentración de $200 \times 10^{6}$ espermatozoides/ $\mathrm{ml}$. Las pajuelas fueron colocadas en atmósfera de vapor de nitrógeno líquido (LN2) de manera horizontal a $4 \mathrm{~cm}$ durante $10 \mathrm{~min}$ y posteriormente sumergidas y almacenadas en nitrógeno líquido $\left(-197^{\circ} \mathrm{C}\right)$ por una semana para su posterior evaluación. Las pajuelas con semen de cada uno de los animales fueron descongeladas en un baño de agua a $37^{\circ} \mathrm{C}$ por $30 \mathrm{seg}$. El semen descongelado fue diluido en TALP a una concentración ajustada a 200 x 106 células/ $\mathrm{ml}$. El mismo volumen de suspensión espermática $(0,5 \mathrm{ml})$ fue procesado por Swim-up y por centrifugación por gradientes Equipure ${ }^{\circledR}$ (Nidacon, International $A B$, Molndal, Sweden), compuesta por: Bottom Layer ${ }^{\circledR}$ (densidad de $80 \%$ ) y Top Layer® (densidad de $40 \%$ ).

Selección espermática. La técnica Swim-up se realizó de acuerdo al procedimiento descrito por Sieme et al. (2003) con algunas modificaciones. Una alícuota de $0,5 \mathrm{ml}$ (volumen inicial) de suspensión espermática descongelada fue colocada en dos tubos $(0,25 \mathrm{ml}$ cada uno) que contenían 1 $\mathrm{ml}$ de medio TALP. Se incubaron por $30 \mathrm{~min}$ a $37^{\circ} \mathrm{C}$, en forma inclinada. Desde la parte superior de cada tubo se extrajeron $0,8 \mathrm{ml}$ de medio, los que fueron mezclados, 
centrifugados a $1000 \mathrm{x}$ g por 10 min y el pellet resuspendido en $0,5 \mathrm{ml}$ (volumen final) de TALP. Para la técnica con Equipure $囚$, en un tubo Falcon de $15 \mathrm{ml}$ se le agregó $2 \mathrm{ml}$ de Bottom Layer® (80\%) y 2 ml Top Layer® (40\%), en la parte superior de la gradiente se depositaron $0,5 \mathrm{ml}$ (volumen inicial) de suspensión espermática descongelada. La gradiente de Equipure $®$ fue centrifugada a $400 \mathrm{x}$ g por 15 min. Después de la centrifugación, el sobrenadante sobre la fracción espermática fue cuidadosamente removido. La fracción de espermatozoides fue diluida en $4 \mathrm{ml}$ de TALP, centrifugada y el pellet resuspendido en $0,5 \mathrm{ml}$ (volumen final) de TALP.

Evaluación función espermática. Post selección se analizó la función espermática, los espermatozoides provenientes de cada uno de los métodos ensayados fueron diluidos en medio TALP, centrifugados a $600 \mathrm{x}$ g por $5 \mathrm{~min}$, se eliminaron los sobrenadantes y los pellets fueron resuspendidos en medio TALP a una concentración de $1 \mathrm{x}$ 106 espermatozoides/ml. Se utilizaron $0,25 \mathrm{ml}$ de suspensión espermática para cada tinción. Los protocolos utilizados para las tinciones por citometría de flujo han sido descritos previamente para espermatozoides de equino.

Viabilidad e integridad de la membrana plasmática. Se utilizó SYBR-14/PI [LIVE/DEAD® Sperm Viability Kit; Molecular Probes Eugene, OR, USA] (Hoogewijs et al., 2010).

Potencial de membrana mitocondrial $(\boldsymbol{\Delta} \Psi \mathrm{m})$. Se utilizó JC-1 (Ortega Ferrusola et al.).

Integridad de la membrana acrosoma. Se utilizó el Kit FITC-PSA/PI [Sigma St. Louis MO, USA] (Salazar et al., 2011). En cada ensayo el análisis de cada tinción fue repetido tres veces.

Citometría de flujo. Las evaluaciones se realizaron en un citómetro de flujo FACScalibur (Becton Dickinson, Mountain View, CA, USA). Las células fueron excitadas por un láser de argón de $488 \mathrm{~nm}$. Cada análisis consistió en la adquisición de un mínimo de 10.000 espermatozoides teñidos, los cuales fueron cuantificados simultáneamente para fluorescencia verde y roja. Se evaluaron 10.000 células/s a través del láser. De acuerdo a parámetros de granularidad relativa (Sidescatter-SSC) y tamaño relativo (Forwardscatter-FSC) las células fueron separadas, de manera que sólo las que poseían características de dispersión de luz típicas de espermatozoides de equino fueron analizadas para fluorescencias. Los datos para los canales de fluorescencia verde y roja fueron obtenidos por escala logarítmica y analizados por medio de un Software BD Cell Quest $^{\mathrm{TM}}$ Pro.
Análisis estadístico. Los análisis estadísticos fueron realizados con el programa GraphPad Prism®, versión 5 (GraphPad Software, San Diego CA). Los datos que se obtuvieron, para los diferentes parámetros funcionales que se evaluó por citometría de flujo, fueron presentados como promedio \pm desviación estándar (DE). El análisis estadístico se aplicaron las pruebas de comparación múltiple de Dunn's y e comparación múltiple de Tukey para datos paramétricos, con las muestras seleccionadas por Swim-up y Equipure ${ }^{\circledR}$ así se determinó diferencias entre los grupos, además una prueba $\mathrm{t}$ para datos paramétricos y no paramétricos para comparar los método por cada animal en las muestras seleccionadas por Swim-up y Equipure®. Las diferencias fueron consideradas significativas para un $\mathrm{P}<0,05$.

\section{RESULTADOS}

La motilidad progresiva obtenida (Promedio \pm DE) de los espermatozoides al comparar SU y EQ en cada uno de los animales, se observó que dos animales presentaron diferencias significativas $(\mathrm{P}<0,05)$ entre ambos métodos, siendo superior la motilidad obtenida por $\mathrm{SU}: \mathrm{A}(55,7 \pm 5,8 \%$ vs. $38,17 \pm 3,7 \%)$ y C $(37,5 \pm 7 \%$ vs. $32 \pm 2,1 \%)$. Sin embargo, en los otros dos no se observaron diferencias significativas (B: $24,2 \pm 3,2 \%$ vs. $21,8 \pm 2,2 \%$ y D: $36,4 \pm 5,3 \%$ vs. $37,5 \pm 5,2 \%$, respectivamente) (Fig. 1a).

Los porcentajes (\%, Promedio \pm DE) de espermatozoides de equinos viables con membrana plasmática intacta al comparar SU y EQ no presentó diferencia significativa $(\mathrm{P}>0,05)$ en un animal $\mathrm{B}(15,2 \pm 6,4$ vs. $12,6 \pm 0,7)$. Sin embargo, se observó diferencia significativa en tres animales, siendo superior SU en dos de ellos (A: $54,3 \pm 1,7$ vs. $36,7 \pm 1,9$, C: $36,1 \pm 5,7$ vs. $29,4 \pm 4,8$ y D: $34,4 \pm 9,4$ vs. $52,7 \pm 5,2$, respectivamente) (Fig. 1b).

Los de espermatozoides de equinos con un alto $\Delta \Psi \mathrm{m}$ (\%, Promedio \pm DE) al comparar SU y EQ se observó en los cuatro animales diferencias significativas $(\mathrm{P}<0,05)$, siendo

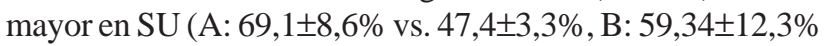
vs. $24,8 \pm 1,5 \%, C: 54,9 \pm 12,3 \%$ vs. $43,2 \pm 3,1 \%$ y D: $53,1 \pm 17,6 \%$ vs. $37,5 \pm 5,7 \%$, respectivamente) (Fig. 1c).

Los porcentajes ( $\%$, Promedio \pm DE) de espermatozoides con la membrana acrosomal intacta al comparar SU y EQ, se observó en los cuatro animales diferencias significativas siendo mayor en dos animales con EQ (A : $32,2 \pm 2,7 \%$ vs. $34,6 \pm 2,7 \%$, B: $12,7 \pm 8,0 \%$ vs. $7,4 \pm 0,9 \%, \mathrm{C}$ : $23,3 \pm 4,1 \%$ vs. $18,2 \pm 2,4 \%$ y D: $14,1 \pm 8,3 \%$ vs. $26,3 \pm 3,1 \%$; $\mathrm{P}<0,05$, respectivamente) (Fig. 1d) 


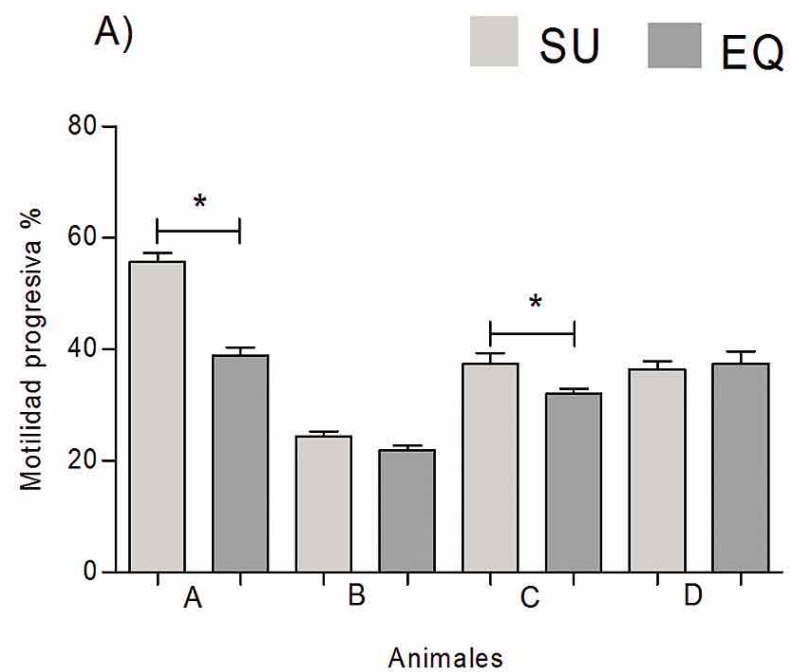

C)

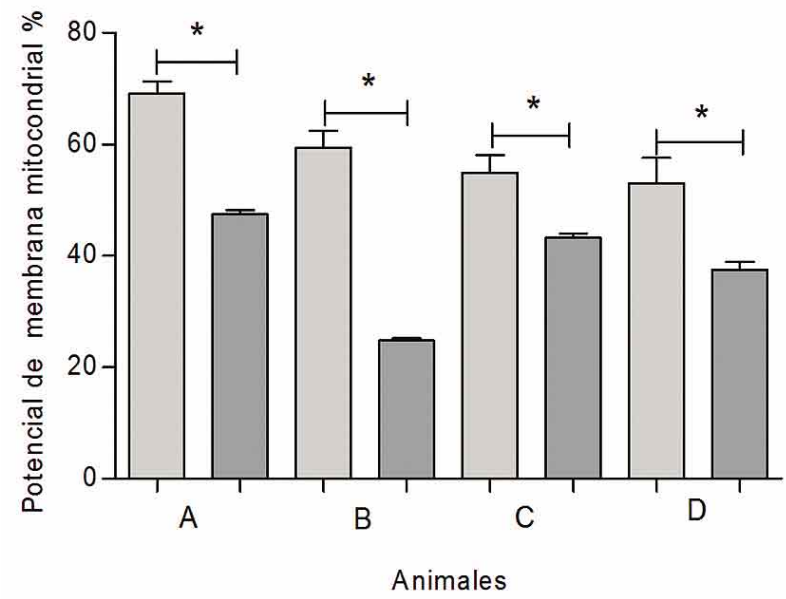

B)

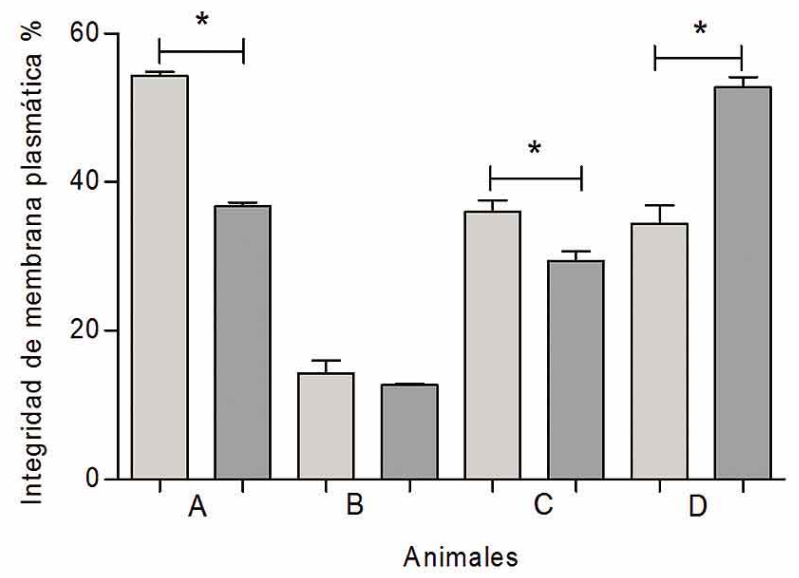

D)

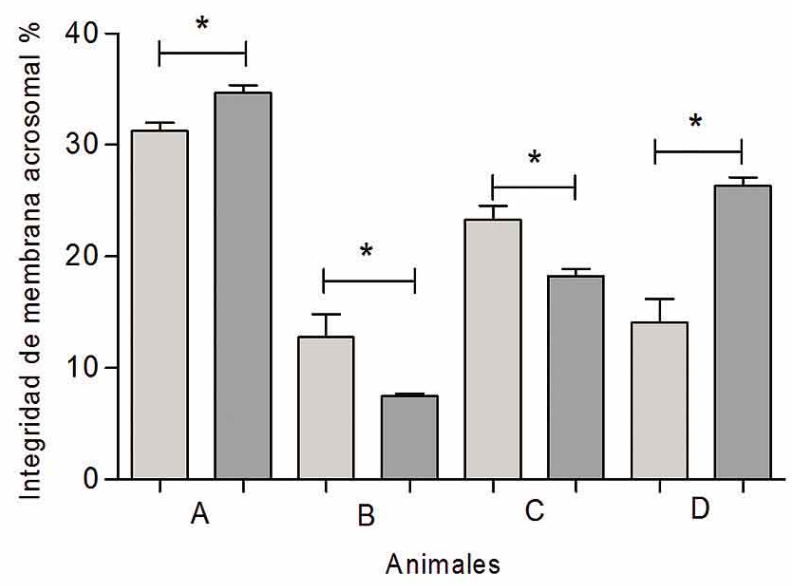

Fig. 1. Porcentaje (Media \pm DE, $n=15$ ) de los diferentes parámetros estudiados: A) Motilidad progresiva, B) Viabilidad e integridad de membrana plasmatica, C) Integridad de membrana mitochondrial, D) Integridad de membraba acrosomal, en espermatozoides de equino descongelados. Los datos fueron agrupados en un gráfico por cada parámetro analizado. * ${ }^{*}$ Indica diferencias significativas $(\mathrm{P}<0.05)$.

\section{DISCUSIÓN}

En equino se ha demostrado que existe un alto grado de variabilidad en la calidad del semen entre los machos e incluso entre los múltiples eyaculados de un mismo animal (Dowsett et al.) como también entre los animales a los procesos de congelación-descongelación (Hoffmann et al.). Además, los procedimientos de congelación y descongelación en los espermatozoides de equino, inducen cambios estructurales y funcionales en los espermatozoides, fragmentación del ADN espermático (Baumber et al.), translocación de la fosfatidilcerina (Thomas et al., 2006), disminución del YDm (Brum et al.), daños en el acrosoma (Atroshchenko \& Bragina, 2011).
El presente estudio evaluó la eficiencia de dos técnicas de selección espermática Swim-up y Equipure® (SU y EQ) en semen descongelado de equino, con respecto a la obtención de una población con mayores porcentajes de espermatozoides con integridad de membrana plasmática, potencial de membrana mitocondrial, integridad de la membrana acrosomal.

Con respecto a la motilidad progresiva de los espermatozoides se observaron diferencias solamente en dos animales (Ay C), las motilidades obtenidas por SU fueron más altas. Sin embargo, en los otros dos animales no se pre- 
sentaron diferencias significativas. Los resultados obtenidos confirman la variabilidad de los espermatozoides de equino y la capacidad de los diferentes métodos en seleccionar los motiles. Cabe señalar que, en investigaciones previas realizadas en bovino con pool de semen, señalan que con SU a pesar de obtener una menor concentración espermática, sin embargo, esta población celular tiene un mayor porcentaje de espermatozoides motiles y con capacidad fecundante comparado con los obtenidos por método de centrifugación por gradiente de densidad como el Percoll ® (Mehmood et al., 2009). No obstante, los resultados concuerdan en que los espermatozoides obtenidos por SU poseen una mayor capacidad fecundante (Parrich et al., 1995). Además, previos trabajos han reportado tasas relativamente altas de fecundación in vitro (FIV) en equino $29 \%$ y $34 \%$ con semen congelado-descongelado, en los cuales se ha utilizado SU como método de selección espermática (Torner et al., 2002).

Similarmente, con respecto a la viabilidad e integridad de membrana plasmática se obtuvieron porcentajes más altos en dos animales (A y C) con SU, y solo en un animal (D) fue superior con EQ, en el otro (B) no hubo diferencia. En investigaciones realizadas en bovino con pool de semen, señalan que con SU, la población celular tiene un mayor porcentaje de espermatozoides viables con membrana plasmática intacta, evaluados por HOST comparado con los obtenidos por método de centrifugación por gradiente de densidad como el Percoll@ (Mehmood et $a l$.). Contrariamente, Somfai et al. (2002) señalan que con Percoll® se obtiene una población espermática de mayor viabilidad comparada con SU. Los resultados obtenidos respecto a este parámetro confirman la variabilidad de los espermatozoides de equino y la capacidad de los diferentes métodos en seleccionar los viables con membrana plasmática intacta.

Con respecto al potencial de membrana mitocondrial en todos los animales, SU fue significativamente más eficiente en recuperar espermatozoides con una alto potencial comparado con EQ. Stoll et al., señalan que en semen congelado/descongelado de equino con EQ optimiza la calidad espermática con respecto al potencial de membrana mitocondrial, obteniendo un porcentaje $(81,6 \pm 1,8 \%)$ más alto de $\Delta \Psi$ m que los logrados $(38,3 \%)$ en el presente trabajo. Esta diferencia puede ser explicada por las razas utilizadas, medios y procedimientos de criopreservación. En equino, a nuestro conocimiento no existen antecedentes sobre la eficiencia de EQ con respecto a SU referente a este parámetro.

La integridad de la membrana acrosomal, es uno de los parámetros más vulnerables en el proceso de con- gelación (Atroshchenko \& Bragina, 2011), en el presente estudio post descongelación y selección, dos animales (A y D) presentaron mayor porcentaje de espermatozoides vivos con acrosoma intacto en EQ, sin embargo, los otros dos (B y C) fue más eficiente en SU. Mehmood et al. en bovinos, no observaron diferencias entre las técnicas de selección Percoll@ y SU con respecto a este parámetro. No obstante, Palomo et al. (1999) en espermatozoides de carnero, observaron una alta integridad de la membrana acrosomal utilizando SU comparado con el Percoll@. Contrariamente, Somfai et al. en espermatozoides de bovino, señalan que Percoll es más eficiente que SU en seleccionar espermatozoides vivos con acrosoma intacto. Los resultados obtenidos en el presente estudio apoyan lo reportado por estudios previos que señalan que en equinos la diferencia entre las técnicas de selección se pueden atribuir a la variabilidad individual (Allen, 2005; Sieme et al., 2008).

Los resultados obtenidos en el presente estudio demostraron que los métodos de selección espermática Swimup y Equipure ${ }^{\circledR}$ permiten recuperar espermatozoides de diferente calidad funcional en semen congelado-descongelado de equino presentándose diferencias individuales con respecto a los métodos. Se observó una tendencia del método Swim-up en seleccionar espermatozoides de equino descongelados con mayor calidad funcional comparado con Equipure®. Es importante considerar la variabilidad entre los animales en la elección del método de selección a utilizar en los procedimientos de asistencia reproductiva.

\section{AGRADECIMIENTOS}

Proyecto DI12-0089 (JR). Dirección de Investigación, Universidad de La Frontera, Temuco, Chile.

CABRERA, P.; SÁNCHEZ, R. \& RISOPATRÓN, J. Sperm selection in frozen/thawed semen of equine: evaluation of plasma, acrosome membranes and mitochondrial membrane potential. Int. J. Morphol., 32(2):725-731, 2014.

SUMMARY: Freeze-thaw procedures induce structural and functional changes in sperm. It is important to use post thaw sperm selection procedures that can retrieve highly functional sperm. The aim of this study was to compare the efficiency of the Swim-up and Equipure ${ }^{\circledR}$ in the selection of functional sperm of thawed equine semen. Semen of four Chilean Criollo reproductive stallions (A, B , C and D) were frozen and thawed using a standard protocol and processed separately $(n=15)$ : I. Swim-up (SU) and II. Equipure ${ }^{\circledR}$ (EQ). Post sperm selection,was determined by flow cytometry. Viability and plasma membrane integrity (SYRB-14/ 
PI), mitochondrial membrane potential (YDm, JC -1), acrosome membrane integrity (FITC-PSA/PI). Progressive motility (\%) was higher $(\mathrm{P}<0.05)$ in two animals per SU compared with EQ, A $(55.7 \pm 5.8 \%$ vs. $38.17 \pm 3.7 \%)$ and $\mathrm{C}(37.5 \pm 7.0 \%$ vs. $32 \pm 2.1 \%$, respectively). The viability and integrity of the plasma membrane $(\%)$, three animals showed differences $(\mathrm{P}<0.05)$, being higher for $\mathrm{SU}$ in two animals compared with EQ (A: $54.3 \pm 1.7$ vs. $36.7 \pm 1.9$, C: $36.1 \pm 5.7$ vs. $29.4 \pm 4.8$ and D: $34.4 \pm 9.4$ vs. $52.7 \pm 5.2$, respectively), only one animal was higher EQ. In YDm (\%), significant differences were detected $(\mathrm{P}<0.05)$ in all four animals, being higher in SU compared with EQ (A: $69.1 \pm 8.6 \%$ vs. $47.4 \pm 3.3 \%$ B: $59.34 \pm 12.3 \%$ vs. $24.8 \pm 1.5 \%$, C: $54.9 \pm 12.3 \%$ vs. $43.2 \pm 3.1 \%$ and D: $53.1 \pm 17.6 \%$ vs. $37.5 \pm 5.7 \%$, respectively). The results obtained in this study showed that sperm selection methods Swim-up and Equipure ${ }^{\circledR}$ can retrieve different functional sperm quality in frozen-thawed equine semen, and that individual differences were registered among animals with respect to methods. In the Swim-up method a tendency for selecting higher functional quality in thawed equine sperm was observed when compared to Equipure ${ }^{\circledR}$.

\section{KEY WORDS: Equine; Sperm; Swim-up; Equipure®}

\section{REFERENCIAS BIBLIOGRÁFICAS}

Allen, W. R. The development and application of the modern reproductive technologies to horse breeding. Reprod Domest Anim., 40(4):310-29, 2005.

Atroshchenko, M. M. \& Bragina, E. E. Change in the ultrastructure of stallion spermatozoa under the effect of cryopreservation. Russ. Agr. Sci., 37(2):175-8, 2011.

Baumber, J.; Ball, B. A.; Linfor, J. J. \& Meyers, S. A. Reactive oxygen species and cryopreservation promote DNA fragmentation in equine spermatozoa. J. Androl., 24(4):621-8, 2003.

Burnaugh, L.; Sabeur, K. \& Ball, B. A. Generation of superoxide anion by equine spermatozoa as detected by dihydroethidium. Theriogenology, 67(3):580-9, 2007.

Brinsko, S. P.; Blanchard, T. L.; Varner, D.; Schumacher, J. \& Love, C. Manual of equine reproduction. 3rd ed. Maryland Heights, Mosby Elsevier, 2011. pp.160-202.

Brum, A. M.; Sabeur, K. \& Ball, B. A. Apoptotic-like changes in equine spermatozoa separated by density-gradient centrifugation or after cryopreservation. Theriogenology, 69(9):1041-55, 2008.

Dowsett, K. F. \& Knott, L. M. The influence of age and breed on stallion semen. Theriogenology, 46(3):397-412, 1996.

Foster, M. L.; Love. C. C.; Varner, D. D.; Brinsko, S. P.; Hinrichs, K.; Teague, S.; Lacaze, K. \& Blanchard, T. L. Comparison of methods for assessing integrity of equine sperm membranes. Theriogenology, 76(2):334-41, 2011.
Hoffmann, N.; Oldenhof, H.; Morandini, C.; Rohn, K. \& Sieme, $\mathrm{H}$. Optimal concentrations of cryoprotective agents for semen from stallions that are classified 'good' or 'poor' for freezing. Anim. Reprod. Sci., 125(1-4):112-8, 2011.

Hoogewijs, M.; Rijisselaere, T.; De Vliegher, S.; Vanhaesebrouck, E.; De Schauwer, C.; Govaere, J.; Thys, M.; Hoflack, G.; Van Soom, A. \& de Kruif, A. Influence of different centrifugation protocols on equine semen preservation. Theriogenology, 74(1):118-26, 2010.

López-Fernández, C.; Crespo, F.; Arroyo, F.; Fernández, J. L.; Arana, P.; Johnston, S. D. \& Gosálvez, J. Dynamics of sperm DNA fragmentation in domestic animals II. The stallion. Theriogenology, 68(9):1240-50, 2007.

Macías García, B.; González Fernández, L.; Ortega Ferrusola, C.; Morillo Rodríguez, A.; Gallardo Bolaños, J. M.; Rodríguez Martinez, H.; Tapia, J. A.; Morcuende, D. \& Peña, F. J. Fatty acids and plasmalogens of the phospholipids of the sperm membranes and their relation with the post-thaw quality of stallion spermatozoa. Theriogenology, 75(5):8118, 2011.

Mehmood, A.; Anwar, M. \& Naqvi, S. M. Motility, acrosome integrity, membrane integrity and oocyte cleavage rate of sperm separated by swim-up or Percoll gradient method from frozen-thawed buffalo semen. Anim. Reprod. Sci., 111(24):141-8, 2009.

Ortega Ferrusola, C.; González Fernández, L.; Salazar Sandoval, C.; Macías García, B.; Rodríguez Martínez, H.; Tapia, J. A. \& Peña, F. J. Inhibition of the mitochondrial permeability transition pore reduces "apoptosis like" changes during cryopreservation of stallion spermatozoa. Theriogenology, 74(3):458-65, 2010.

Palomo, M. J.; Izquierdo, D.; Mogas, T. \& Paramio, M. T. Effect of semen preparation on IVF of prepubertal goat oocytes. Theriogenology, 51(5):927-40, 1999.

Parrish, J. J.; Krogenaes, A. \& Susko-Parrish, J. L. Effect of bovine sperm separation by either swim-up or Percoll method on success of in vitro fertilization and early embryonic development. Theriogenology, 44(6):859-69, 1995.

Pojprasath, T.; Lohachit, C.; Techakumphu, M.; Stout, T. \& Tharasanit, T. Improved cryopreservability of stallion sperm using a sorbitol-based freezing extender. Theriogenology, 7(9):1742-9, 2011.

Salazar, J. L. Jr.; Teague, S. R.; Love, C. C.; Brinsko. S. P.; Blanchard, T. L. \& Varner, D. D. Effect of cryopreservation protocol on postthaw characteristics of stallion sperm. Theriogenology, 76(3):409-18, 2011.

Schober, D.; Aurich, C.; Noh, H. \& H. Gille, L. Influence of cryopreservation on mitochondrial functions in equine spermatozoa. Theriogenology, 68(5):745-54, 2007. 
CABRERA, P.; SÁNCHEZ, R. \& RISOPATRóN, J. Selección espermática en semen congelado/descongelado de equino: evaluación de las membranas plasmática, acrosomal y potencial de membrana mitocondrial. Int. J. Morphol., 32(2):725-731, 2014.

Sieme, H.; Martinsson, G.; Rauterberg, H.; Walter, K.; Aurich, C.; Petzoldt, R. \& Klug, E. Application of techniques for sperm selection in fresh and frozen-thawed stallion semen. Reprod. Domest. Anim., 38(2):143-40, 2003.

Sieme, H.; Harrison, R. A. \& Petrunkina, A. M. Cryobiological determinants of frozen semen quality, with special reference to stallion. Anim. Reprod. Sci., 107(3-4):276-92, 2008.

Somfai, T.; Bodó, S.; Nagy, S.; Papp, A. B.; Iváncsics, J.; Baranyai, B.; Gócza, E. \& Kovács, A. Effect of swim up and Percoll treatment on viability and acrosome integrity of frozen-thawed bull spermatozoa. Reprod. Domest. Anim., 37(5):285-90, 2002.

Stoll, A.; Loce, C. C. \& Ball, B. A. Use of a Single-Layer Density Centrifugation Method Enhances Sperm Quality in Cryopreserved-Thawed Equine Spermatozoa. J. Equine Vet. Sci., 33(7):547-51, 2012.

Stuhtmann, G.; Oldenhof, H.; Peters, P.; Klewitz, J.; Martinsson, G. \& Sieme, H. Iodixanol density gradient centrifugation for selecting stallion sperm for cold storage and cryopreservation. Anim. Reprod. Sci., 133(3-4):184-90, 2012.

Torner, H.; Alm, H.; Mlodawska, W.; Warnke, C.; Göllnitz, K.; Blottner, S. \& A. Okolski, A. Determination of development in horse zygotes and spermatozoa during fertilization in vitro. Theriogenology, 58(2-4):693-6, 2002.

Thomas, A. D.; Meyers, S. A. \& Ball, B. A. Capacitation-like changes in equine spermatozoa following cryopreservation. Theriogenology, 65(8):1531-50, 2006.

\author{
Dirección para Correspondencia: \\ Paulina Cabrera Herreros \\ Centro de Biotecnología en Reproducción \\ Facultad de Medicina \\ Universidad de La Frontera \\ Temuco \\ CHILE
}

Email: polinc16@live.com

Recibido: 30-01-2014

Aceptado: 23-05-2014 